PEST MANAGEMENT

\title{
Levantamento Populacional de Cigarrinhas (Hemiptera: Cicadellidae) Associadas à Transmissão de Xylella fastidiosa em Pomares Cítricos do Litoral Norte da Bahia
}

\author{
Marcelo P De Miranda ${ }^{1}$, João R S Lopes ${ }^{1}$, Antonio S Do Nascimento ${ }^{2}$, José L Dos Santos 3 , \\ RODNEY R CAVICHIOLI ${ }^{4}$
}

${ }^{1}$ Depto. Entomologia, Fitopatologia e Zoologia Agrícola, ESALQ/USP, C. postal 9, 13418-900, Piracicaba, SP; mmiranda@esalq.usp.br; ${ }^{2}$ Depto.Entomologia, EMBRAPA/CNPMF, C. postal 7, 44380-000, Cruz das Almas, BA; antnasc@cnpmf.embrapa.br; ${ }^{3}$ Escritório Regional,EBDA, 48330-000,Rio Real, BA; ebdaproreal@terral.com.br; ${ }^{4}$ Depto. Zoologia, Univ. Federal do Paraná. C. postal 19020,81531-980 Curitiba, PR; Bolsista do CNPq; cavich@ufpr.br

Edited by André L Lourenção - IAC

Neotropical Entomology 38(6):827-833 (2009)

Survey of Sharpshooters (Hemiptera: Cicadellidae) Associated with Xylella fastidiosa Transmission in Citrus Groves of the North Coast of Bahia State

\begin{abstract}
The causal agent of citrus variegated clorosis, Xylella fastidiosa, is transmitted by leafhoppers of the subfamily Cicadellinae, whose species vary regionally. The goal of this study was to identify potential vectors of this pathogen in citrus groves of Bahia North Coast, Brazil. The survey was done from March/2002 to February/2003 in three seven- to nine-year-old sweet orange (Citrus sinensis, Pêra variety) groves located in Rio Real, BA. Fifteen yellow sticky cards $(8.5 \times 11.5 \mathrm{~cm})$ were installed $40 \mathrm{~m}$ apart in each grove, hanged at $1.5 \mathrm{~m}$ high on the upper north side of citrus canopies, and replaced fortnightly. A sweep net was periodically used to sample leafhoppers on herbaceous weeds inside the groves, by selecting five points at random and performing 30 sweeps in each point. A total of 1,360 specimens of 49 Auchenorrhyncha species were collected in this study, mostly in the family Cicadellidae (90.2\%). The subfamily Cicadellinae, which includes the sharpshooter vectors of $X$. fastidiosa, showed the largest number of species (14) and specimens (84.8\%). Acrogonia flagellata Young, A. citrina Marucci \& Cavichioli, Homalodisca spottii Takiya, Cavichioli \& McKamey and an unidentified Cicadellini (species 1) were the dominant species trapped on citrus canopies, while Hortensia similis (Walker) and Erythrogonia dubia (Medler) were dominant in the weedy vegetation. Among the Cicadellinae species already known as vectors of $X$. fastidiosa in citrus, only A. citrina, Bucephalogonia xanthophis (Berg) e Ferrariana trivittata (Signoret) were found. The two latter species were accidentally trapped by sweep net in the weedy vegetation.
\end{abstract}

KEY WORDS: Citrus variegated chlorosis, sharspshooter vector, Cicadellinae, species composition

RESUMO - O agente causal da clorose variegada dos citros, Xylella fastidiosa, é transmitido por cigarrinhas da subfamília Cicadellinae, cujas espécies variam regionalmente. O objetivo deste trabalho foi identificar potenciais vetores do patógeno em pomares cítricos do Litoral Norte da Bahia. Os levantamentos foram conduzidos de mar/2002 a fev/2003, em três propriedades no município de Rio Real, em pomares de laranjeira doce (Citrus sinensis) variedade Pêra, com sete a nove anos. Em cada pomar, 15 cartões adesivos amarelos foram dispostos a $40 \mathrm{~m}$ entre si, instalados a $1,5 \mathrm{~m}$ de altura em ramos da face norte, na periferia da copa, sendo substituídos quinzenalmente. Também foram realizadas amostragens com rede de varredura na vegetação rasteira dos pomares, em cinco pontos escolhidos aleatoriamente, efetuando-se 30 redadas por ponto. No total, foram coletados 1360 espécimes pertencentes a 49 espécies de Auchenorrhyncha, a maioria da família Cicadellidae (90,2\%). A subfamília Cicadellinae, que inclui os vetores de $X$. fastidiosa, apresentou maior número de espécies (14) e de indivíduos coletados (84,8\%). Entre as espécies de Cicadellinae, Acrogonia flagellata Young, A citrina Marucci \& Cavichioli, Homalodisca spottii Takiya, Cavichioli \& McKamey e uma espécie não-identificada de Cicadellini (espécie 1) foram dominantes na copa das plantas cítricas, enquanto que Hortensia similis (Walker) e Erythrogonia dubia (Medler) foram dominantes na vegetação rasteira. Entre os cicadelíneos já conhecidos como vetores de $X$. fastidiosa em citros, apenas $A$. citrina, 
Bucephalogonia xanthophis (Berg) e Ferrariana trivittata (Signoret) foram observados, sendo que as duas últimas espécies tiveram ocorrência acidental na vegetação rasteira.

PALAVRAS-CHAVE: Clorose variegada dos citros, vetor, Cicadellinae, A. citrina, diversidade

A clorose variegada dos citros (CVC) é um dos problemas fitossanitários de maior relevância para a citricultura brasileira e é causada por uma bactéria Gramnegativa, Xylella fastidiosa, limitada aos vasos do xilema das plantas (Lee et al 1993). Detectada inicialmente no estado de São Paulo, em 1987, a CVC só foi constatada no Nordeste do Brasil em 1996, no município de Boquim, SE, e posteriormente na Bahia, em pomares comerciais dos municípios de Rio Real e Itapicuru (De Negri 1990, Santos Filho et al 1999).

Os insetos relatados como vetores de $X$. fastidiosa são cigarrinhas pertencentes às famílias Cicadellidae (subfamília Cicadellinae) e Cercopidae, que se alimentam do xilema das plantas (Purcell 1989). Diversos Cicadellinae ocorrem em pomares cítricos no estado de São Paulo (Paiva et al 1996, Yamamoto \& Gravena 2000), sendo que vários deles já foram confirmados como vetores desse patógeno (Roberto et al 1996, Yamamoto et al 2002).

Dada a importância das cigarrinhas na disseminação da CVC, a identificação das espécies vetoras predominantes em pomares cítricos e o conhecimento de sua distribuição ao longo do ano são informações fundamentais para o manejo da doença (Lopes 1999, Yamamoto \& Gravena 2000). Estudos de interações desses vetores com a planta cítrica, vegetação rasteira de pomares e fatores climáticos, desenvolvidos principalmente no estado de São Paulo, embasaram estratégias de manejo que atualmente permitem a convivência com a CVC (Yamamoto \& Lopes 2004).

Tendo em vista as peculariedades edafo-climáticas e de vegetação de cada região do Brasil, os estudos com vetores realizados na Região Sudeste não são diretamente aplicáveis ao manejo da CVC no Litoral Norte da Bahia, devido às possíveis diferenças na diversidade e abundância de espécies de cigarrinhas vetoras entre as duas regiões. Portanto, este trabalho teve por objetivo identificar espécies de cigarrinhas vetoras ou potenciais vetoras de $X$. fastidiosa em pomares cítricos do Litoral Norte da Bahia e, por meio da análise faunística, selecionar as espécies predominantes com ênfase na subfamília Cicadellinae.

\section{Material e Métodos}

O levantamento foi conduzido em três pomares de laranjeira doce (Citrus sinensis) variedade Pêra, enxertada sobre limoeiro cravo (Citrus limonia) com idade variando de sete a nove anos, localizados nas seguintes propriedades do município de Rio Real, BA: Fazenda Esperança

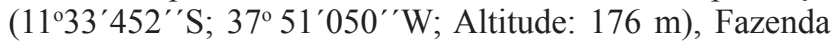
Araújo (11 $31^{\circ} 801^{\prime \prime} \mathrm{S}$; $37^{\circ} 54^{\prime} 518^{\prime \prime} \mathrm{W}$; Altitude: $\left.175 \mathrm{~m}\right)$

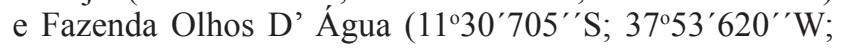
Altitude: $175 \mathrm{~m})$. Essas propriedades estavam inseridas no programa de Produção Integrada de Citros (PIC) e realizavam os mesmos tratos culturais, adubação e controle fitossanitário. Em nenhum local foi realizado controle específico para cigarrinhas, somente pulverizações para Phyllocoptruta oleivora (Asmead) (ácaro da ferrugem), Orthezia praelonga Douglas (cochonillha-de-placas) e Phyllocnistis citrella Stainton (larva minadora).

O clima no Litoral Norte da Bahia, segundo classificação de Thornthwaite, varia entre subúmido a seco e úmido, com precipitação média anual de $949,9 \mathrm{~mm}$ e temperatura média anual de $23,9^{\circ} \mathrm{C}$ (SEI 2006). Os pomares estudados apresentavam em seu entorno um fragmento florestal de floresta estacional semi-decidual à distância de 5-10 m. A vegetação rasteira presente nas entrelinhas dos pomares, em sua maioria, era composta por gramíneas (Cenchrus echinatus, Brachiaria spp., Paspalum spp.) e herbáceas das famílias Commelinaceae (Commelina nudiflora), Solanaceae (Solanum sp.), Amaranthaceae (Amaranthus sp.) e Euphorbiaceae (Euphorbia spp.).

Amostragem. As coletas de cigarrinhas foram realizadas quinzenalmente no período de março de 2002 a fevereiro de 2003, utilizando-se cartões adesivos amarelos de 8,5 x $11,5 \mathrm{~cm}$ (Biocontrole ${ }^{\circledR}$, São Paulo, SP). Em cada pomar, foram instalados 15 cartões adesivos a $1,5 \mathrm{~m}$ de altura do solo, distanciados $40 \mathrm{~m}$ um do outro. Os cartões foram instalados na periferia da copa das plantas cítricas, em ramos da face norte. Periodicamente os cartões foram remanejados para as extremidades dos ramos, e as folhas em sua volta foram removidas com o intuito de não prejudicar a sua atratividade. Foram realizadas, também, amostragens mensais de cigarrinhas na vegetação rasteira das entrelinhas dos pomares, por meio de rede de varredura. Em cada avaliação, amostraram-se cinco pontos escolhidos aleatoriamente, efetuando-se 30 redadas por ponto (Purcell 1994, Yamamoto et al 2001).

Triagem e identificação do material de campo. As cigarrinhas coletadas com rede de varredura foram separadas dos detritos vegetais e de outros insetos, enquanto que as cigarrinhas capturadas por cartões adesivos foram removidas com auxílio de um pincel embebido em querosene. Todas as cigarrinhas coletadas foram contadas e separadas por espécie, local, data e método de coleta, sendo posteriormente acondicionadas em frascos etiquetados, contendo álcool a $70 \%$.

Estabeleceu-se, também, uma coleção de referência onde as cigarrinhas foram separadas e numeradas por espécie. A identificação taxonômica dos espécimes foi realizada com base em caracteres morfológicos das asas (tipo, forma e venação), cabeça (coroa e posição dos ocelos), forma geral do corpo e estruturas genitais de ambos os sexos. Utilizaram-se, ainda, caracteres das estruturas da cápsula genital do macho (estilos, edeago, conectivo, placas, pigóforo) e apódemas 
na porção anterior do abdome (Young 1968, 1977, Nielson 1985). Para os espécimes identificados ao nível de família e subfamília, adotou-se o procedimento de classificação em morfoespécies, com o objetivo de englobar toda a comunidade de cigarrinhas da subordem Auchenorrhyncha nas análises faunísticas.

Análise dos dados do levantamento. Os dados de coleta de cigarrinhas com cartões adesivos e rede entomológica em cada pomar foram submetidos a análise faunística. Baseando-se nos índices de constância, frequência, abundância e dominância, foram determinadas as espécies predominantes, ou seja, aquelas que apresentaram os maiores índices faunísticos (Silveira Neto et al 1995). Para comparação das comunidades de cigarrinhas entre pomares, foram calculados os índices de diversidade de Shannon e Weiner (H'), equitabilidade (E) e similaridade. A comparação estatística dos índices de diversidade e similaridade entre as fazendas avaliadas foi realizada por meio do intervalo de confiança da média a 5\% de probabilidade. Para esse cálculo utilizou-se a fórmula: IC $=\mathrm{m} \pm$ t.s $(\mathrm{m})$, onde: $\mathrm{m}=$ estimativa da média; $\mathrm{t}=2 ; \mathrm{s}(\mathrm{m})$ = erro padrão da média (Mountford 1962, Silveira Neto et al 1976).

Com exceção da similaridade, todos os índices foram calculados através do software ANAFAU (Setor de Entomologia, ESALQ/USP). Os dados de espécies discrepantes foram analisados por meio de análise gráfica de resíduo (Atkinson 1985), permitindo a classificação das mesmas em categorias distintas, denominadas super dominantes, super abundantes e/ou super frequentes.

\section{Resultados}

Nos pomares das três fazendas estudadas, realizaram-se 20 avaliações quinzenais com cartões adesivos amarelos e 13 mensais com rede de varredura, coletando-se 1360 espécimes de Auchenorrhyncha, distribuídos em seis famílias e 49 espécies. As famílias com maior número de espécimes coletados foram Cicadellidae (90,2\%) e Membracidae (9,2\%). As demais famílias (Cercopidae, Cicadidae, Aetalionidae e Derbidae) representaram apenas $0,7 \%$ do total coletado.

Fazenda Olhos D'Água. Nas amostragens com cartões adesivos amarelos, coletou-se o total de 670 espécimes, distribuídos em quatro famílias e 21 espécies (ver as principais em Arquivo Suplementar 1 online). As análises indicaram cinco espécies predominantes, as quais foram classificadas como constantes, dominantes, muito abundantes e muito frequentes. Tais espécies foram: Homalodisca spottii Takiya, Cavichioli \& McKamey com 44,9\% dos espécimes coletados, Cicadellini sp. 1 (19,3\%), Acrogonia citrina Marucci \& Cavichioli (10,9\%), Acrogonia flagellata Young (10,3\%) e Scaphytopius sp. (8,4\%). Ceresa ustulata Fairmaire foi dominante, muito abundante e muito frequente, mas inconstante. Sete espécies de ocorrência acidental foram classificadas ao nível de morfoespécie: três Gyponinae, um Nirvaninae, um Cicadidae e dois Membracidae. Cicadellidae foi a que mais se destacou quanto à porcentagem de indivíduos coletados nos cartões adesivos $(96,6 \%)$, sendo que a subfamília Cicadellinae representou $89,2 \%$ do total de cicadelídeos.

Nas amostragens com rede de varredura na vegetação rasteira do pomar, coletou-se o total de 75 espécimes, distribuídos em duas famílias e nove espécies (ver as principais em Arquivo Suplementar 2 online). Acinopterus gentilis (Berg) e Empoasca sp. destacaram-se em relação às demais, apresentando ambas frequência de 25,3\%. A família Cicadellidae foi a que apresentou a maior porcentagem de espécimes $(74,7 \%)$, com destaque para a subfamília Typhlocybinae, que apresentou $46,5 \%$ do total de cicadelídeos coletados. Nessa subfamília além de Empoasca sp., coletouse outra espécie de ocorrência acidental e classificada ao nível de morfoespécie. Na subfamília Cicadellinae, Hortensia similis (Walker) apresentou o maior número de espécimes $(88,9 \%)$.

Fazenda Araújo. Nas avaliações com cartões adesivos amarelos, coletou-se o total de 192 espécimes, distribuídos em três famílias e 20 espécies (ver as principais em Arquivo Suplementar 1 online). Pela análise faunística, quatro espécies foram predominantes, sendo classificadas como constantes, dominantes, muito abundantes e muito frequentes. Tais espécies foram: Cicadellini sp. 1 (25,5\%); $A$. flagellata (24,5\%); H. spottii. (17,2\%) e C. ustulata $(10,9 \%)$. Outras quatro espécies tiveram ocorrência acidental e foram classificadas ao nível de morfoespécie: dois Agalliinae, um Gyponinae, e um Typhlocybinae. Cicadellidae foi a família mais representativa, com $83,9 \%$ dos indivíduos, sendo que a subfamília Cicadellinae apresentou 90,7\% desse total. As espécies de Cicadellinae consideradas predominantes representaram $67,2 \%$ do total de espécimes de Auchenorrhyncha coletados.

Com rede de varredura, coletaram-se 103 espécimes na vegetação rasteira, distribuídos em três famílias e 11 espécies (ver as principais em Arquivo Suplementar 2 online). Observaram-se duas espécies predominantes, $H$. similis (42,7\%) e C. ustulata (26,2\%). A espécie Empoasca sp. foi dominante, muito abundante e muito frequente, porém não foi constante. Quatro espécies tiveram ocorrência acidental e foram classificadas ao nível de morfoespécie: um Agalliinae, um Deltocephalinae, um Gyponinae e um Typhlocybinae. Cicadellidae foi a família que mais se destacou, com $71,8 \%$ dos indivíduos, sendo que a subfamília Cicadellinae apresentou $68,9 \%$ deste total.

Fazenda Esperança. No levantamento com cartões adesivos amarelos, coletaram-se 256 espécimes, distribuídos em três famílias e 22 espécies (ver as principais em Arquivo Suplementar 1 online). Determinaram-se cinco espécies predominantes, Scaphytopius sp. (19,1\%); A. flagellata (18,0\%); Cicadellini sp. 1 (16,0\%); H. spottii $(14,5 \%)$ e Bahita sp. $1(11,3 \%)$, as quais foram classificadas como constantes, dominantes, muito abundantes e muito frequentes. Outras cinco espécies foram classificadas ao nível de morfoespécie e de ocorrência acidental, sendo: um Agalliinae, um Deltocephalinae, um Nirvaninae e dois 
Membracidae. Cicadellidae foi a família mais representativa quanto à porcentagem de indivíduos capturados $(88,7 \%)$, com destaque para a subfamília Cicadellinae que apresentou 59,0\% deste total de cicadelídeos. As três espécies de Cicadellinae consideradas predominantes, A. flagellata, Cicadellini sp.1 e $H$. spottii, representaram $48,4 \%$ do total de espécimes de Auchenorrhyncha coletados.

$\mathrm{Na}$ vegetação rasteira, com rede de varredura, coletaramse 64 espécimes, distribuídos em três famílias e oito espécies (ver as principais em Arquivo Suplementar 2 online). Nesse levantamento apenas a espécie $H$. similis $(28,1 \%)$ foi predominante, sendo também classificada como constante, dominante, muito abundante e muito frequente. Empoasca sp. não foi constante, embora tenha apresentado elevados índices de dominância, abundância e frequência. Duas espécies, uma de Gyponinae e outra de Debidae tiveram ocorrência acidental e foram classificadas ao nível de morfoespécie. Cicadellidae foi a família mais representativa em relação ao número de indivíduos coletados $(95,3 \%)$. A subfamília Cicadellinae foi a mais numerosa, apresentando $50,8 \%$ do total de cicadelídeos coletados.

Diversidade, equitabilidade e similaridade. A diversidade de espécies de cigarrinhas coletadas por cartões adesivos e rede de varredura não diferiu para os pomares avaliados (Tabela 1). Comparando-se a comunidade de cigarrinhas coletadas por meio de cartões adesivos, a maior similaridade $(8,69 \%)$ foi encontrada entre os pomares das fazendas Olhos D'Água e Esperança (Tabela 2), indicando que entre essas propriedades ocorreu maior número de espécies comuns. Esses pomares também apresentaram o maior número de espécies coletadas. Níveis inferiores de similaridade foram observados entre pomares de laranja nas demais combinações avaliadas. Para a comunidade de cigarrinhas coletadas com rede de varredura, os pomares avaliados apresentaram valores de similaridade semelhantes nas comparações entre as fazendas (Tabela 2). Comparando-se as cigarrinhas capturadas nos dois métodos de coleta, apenas seis espécies foram comuns aos dois métodos utilizados: Agalliinae sp. 2, Cyphonia clavigera (Fabricius), C. ustulata, Erythrogonia dubia Medler, H. spottii, Typhlocybinae sp.

\section{Discussão}

O complexo de cigarrinhas encontrado nos pomares cítricos do Litoral Norte da Bahia pode ser dividido em dois grupos. O primeiro formado por espécies capturadas por cartões adesivos amarelos posicionados a $1,5 \mathrm{~m}$ de altura, as quais possuem atividades principalmente na copa das plantas cítricas. O segundo grupo formado por cigarrinhas que habitam as plantas invasoras presentes nesses pomares, coletadas por meio de rede de varredura. Em ambos os grupos, a subfamília Cicadellinae foi a mais representativa, tanto em diversidade como em abundância. Resultados semelhantes foram observados em pomares cítricos do estado de São Paulo, onde essa subfamília se destacou em relação ao número de espécies e frequência de espécimes coletados com succionador motorizado em plantas cítricas (Yamamoto \& Gravena 2000). A composição da fauna, com relação a famílias e subfamílias de Auchenorrhyncha encontradas nesse hábitat, foi semelhante à observada em São Paulo por Yamamoto \& Gravena (2000).

A ocorrência de grupos distintos de cigarrinhas em plantas cítricas e na vegetação rasteira já havia sido relatada em pomares paulistas (Paiva et al 1996). Entretanto, a composição de espécies de cicadelíneos relatada na copa das plantas cítricas no Sul e Sudeste de São Paulo difere daquela relatada no presente trabalho. Em pomares do Norte do estado de São Paulo predominaram os cicadelíneos Acrogonia sp., Dilobopterus costalimai Young e Oncometopia facialis (Signoret) na copa das plantas cítricas (Paiva et al 1996, Yamamoto \& Gravena 2000). Resultados semelhantes foram obtidos no Noroeste do Paraná, onde as espécies mais representativas foram D. costalimai e A. citrina (Nunes et al 2007). Entre essas espécies arborícolas, que já foram confirmadas como vetoras de $X$. fastidiosa (Roberto et al 1996) e que são consideradas importantes para a disseminação da CVC (Lopes 1999), apenas A. citrina foi coletada por cartões adesivos nos pomares cítricos da Bahia, sendo este o primeiro relato de sua ocorrência no Nordeste. Essa espécie vetora foi encontrada nos três pomares estudados, predominando no da fazenda Olhos D'água. Assim, é provável que a espécie tenha um papel relevante na disseminação da CVC também

Tabela 1 Total de indivíduos, diversidade e equitabilidade de espécies de cigarrinhas capturadas por cartões adesivos amarelos e rede de varredura em pomares cítricos de três fazendas de Rio Real, BA, de março/2002 a fevereiro/2003.

\begin{tabular}{|c|c|c|c|c|c|}
\hline Métodos de captura & Fazendas & $\begin{array}{l}\mathrm{N}^{\mathrm{o}} \text { de } \\
\text { indivíduos }\end{array}$ & $\begin{array}{c}\mathrm{N}^{\mathrm{o}} \mathrm{de} \\
\text { espécies }\end{array}$ & $\begin{array}{c}\text { Diversidade }^{2} \\
\text { Shannon-Wiener (H') }\end{array}$ & Equitabilidade (E) \\
\hline \multirow[t]{3}{*}{ Cartões adesivos } & Olhos D’Água & 670 & 21 & $1,69 \mathrm{a}$ & 0,55 \\
\hline & Araújo & 192 & 20 & $2,09 \mathrm{a}$ & 0,69 \\
\hline & Esperança & 256 & 22 & $2,31 \mathrm{a}$ & 0,74 \\
\hline \multirow[t]{3}{*}{ Rede de varredura } & Olhos D'Água & 75 & 9 & $1,84 \mathrm{a}$ & 0,84 \\
\hline & Araújo & 103 & 11 & $1,67 \mathrm{a}$ & 0,69 \\
\hline & Esperança & 64 & 8 & $1,61 \mathrm{a}$ & 0,77 \\
\hline
\end{tabular}

\footnotetext{
${ }^{1}$ Total coletado em 20 amostragens quinzenais e 13 amostragens mensais por cartões adesivos e de rede de varredura, respectivamente.

${ }^{2}$ Índices seguidos da mesma letra não diferem entre si, dentro de cada método de amostragem, pelo intervalo de confiança a $5 \%$ de probabilidade.
} 
Tabela 2 Índice de similaridade de espécies de cigarrinhas coletadas por rede de varredura e cartões adesivos em pomares cítricos, comparando-se fazendas distintas em Rio Real, BA. Março/2002 a fevereiro/2003.

\begin{tabular}{lcc}
\hline \multirow{2}{*}{ Fazendas comparadas } & \multicolumn{2}{c}{ Similaridade (\%) } \\
\cline { 2 - 3 } & Cartões adesivos & Rede varredura \\
\hline Olhos D’ Água x Araújo & $5,65 \mathrm{~b}$ & $6,78 \mathrm{a}$ \\
Olhos D’ Água x Esperança & $8,69 \mathrm{a}$ & $6,45 \mathrm{a}$ \\
Araújo x Esperança & $5,26 \mathrm{~b}$ & $5,04 \mathrm{a}$ \\
\hline
\end{tabular}

1'́ndices seguidos da mesma letra não diferem entre si na mesma coluna, pelo intervalo de confiança a 5\% de probabilidade.

em pomares cítricos do Nordeste.

Além de $A$. citrina, o presente estudo mostrou que outras três espécies de cicadelíneos são dominantes na copa das laranjeiras na Bahia: $H$. spottii, Cicadellini sp. 1 e $A$. flagellata. A. flagellata havia sido relatada apenas na Guiana Francesa (Young 1968), enquanto que as duas primeiras espécies são novas; a morfoespécie Cicadellini sp.1 ainda está sendo descrita, possivelmente dentro de um novo gênero em Cicadellini (R R Cavichioli, comunicação pessoal). A capacidade de transmissão de $X$. fastidiosa por essas três espécies previamente desconhecidas na citricultura ainda não foi avaliada. Entretanto, elas apresentam características ecológicas que as tornam relevantes como potenciais vetoras de $X$. fastidiosa em citros, tais como abundância, constância, hábito de se alimentar e, possivelmente, reproduzir em laranjeiras (Purcell 1994, Lopes 1999). Essas características são de grande importância no que diz respeito à epidemiologia da CVC, em que a disseminação secundária entre plantas cítricas dentro do pomar é muito importante (Laranjeira et al 1998). O método de captura por armadilhas adesivas não permite inferir a atividade alimentar das cigarrinhas; porém, as mesmas foram observadas em posição de alimentação sobre ramos de plantas cítricas durante a condução do presente estudo (M P de Miranda, comunicação pessoal).

Entre os cicadelíneos que ocorrem na vegetação espontânea dos pomares da Bahia, a espécie $H$. similis destaca-se pela sua maior abundância, frequência e constância, sendo dominante nas três propriedades avaliadas. A espécie também foi a mais frequente e uma das poucas contantes, juntamente com Macugonalia leucomelas (Walker), na vegetação herbácea de um pomar cítrico no Rio Grande do Sul (Ott et al 2006). A predominância de $H$. similis em regiões distantes no Brasil é consistente com informações existentes na literatura, que indicam sua ampla distribuição geográfica no continente americano, especialmente em gramíneas (Young 1977, Wilson \& Claridge 1991). A despeito de sua abundância e ampla distribuição, ainda não há informações sobre sua capacidade de transmitir $X$. fastidiosa para plantas cítricas.

Das espécies previamente relatadas como vetoras de $X$. fastidiosa em citros (Redak et al 2004), apenas Ferrariana trivittata (Signoret) e Bucephalogonia xanthophis (Berg) foram coletadas na vegetação rasteira dos pomares da Bahia, sendo ambas de ocorrência acidental. No estado de São Paulo, F. trivittata é abundante em gramíneas da vegetação rasteira
(Paiva et al 1996), enquanto que B. xanthophis é o cicadelíneo mais frequente em viveiros cítricos abertos (Roberto et al 2000) ou pomares em formação (Yamamoto et al 2001). Algumas plantas invasoras presentes em pomares cítricos podem hospedar $X$. fastidiosa; no entanto, não há evidências de que as mesmas sejam importantes como fontes de inóculo do patógeno para aquisição pelas cigarrinhas e posterior transmissão para plantas cítricas (Lopes et al 2003). Além disso, as cigarrinhas predominantes nesse tipo de vegetação raramente são observadas nas plantas cítricas ou vice-versa (Paiva et al 1996). No presente trabalho, apenas duas espécies de cicadelíneos capturadas na copa das laranjeiras (E. dubia e $H$. spottii) foram observadas na vegetação rasteira. A ocorrência acidental de $H$. spottii nesse segundo hábitat (apenas um espécime capturado) é indicativo de que se trata de uma espécie predominantemente arborícola. Erythrogonia dubia foi acidental em ambos os hábitats.

É interessante salientar que o cicadelídeo Scaphytopius sp. (subfamília Deltocephalinae) foi abundantemente coletado por cartões adesivos amarelos em quase todas as áreas avaliadas. Espécies desse gênero são descritas como vetoras do molicute Spiroplasma citri, agente causal da doença conhecida como stubborn dos citros (Kaloostian et al 1979), que ainda não foi relatada no Brasil. Assim, o conhecimento das cigarrinhas desse grupo também é importante, pois caso ocorra a introdução deste patógeno, os aspectos epidemiológicos relacionados à sua transmissão serão mais facilmente compreendidos. Recentemente, detectou-se a presença de um fitoplasma associado a uma doença com sintomas semelhante ao greening (ou huanglongbing) em plantas cítricas no estado de São Paulo (Teixeira et al 2008), sendo as cigarrinhas da subfamília Deltocephalinae e os psilídeos os potenciais vetores desse patógeno. Além de Scaphytopius sp., deltocefalíneos do gênero Bahita também ocorreram em números significativos em armadilhas adesivas de uma das áreas avaliadas na Bahia.

A ausência de diferenças entre os pomares avaliados com relação à diversidade, provavelmente ocorreu pelo fato de os mesmos apresentarem semelhanças no tipo de vegetação adjacente e na faixa etária (7-9 anos), além de estarem localizados em uma mesma região produtora. Giustolin et al (2009) também observaram semelhanças na diversidade de cigarrinhas coletadas em pomares cítricos do estado de São Paulo, que apresentavam características similares entre si e estavam situados próximos ao mesmo tipo de fragmento florestal. Nesse caso a vegetação adjacente aos pomares poderia estar funcionando como reservatório de espécies, favorecendo um possível fluxo de cigarrinhas entre os dois ambientes e, consequentemente, a maior diversidade nos pomares. Assim, a presença do fragmento florestal ao lado dos pomares avaliados no Litoral Norte da Bahia pode ter sido um dos principais fatores que contribuíram para os semelhantes índices de diversidade observados entre estes.

A menor equitabilidade observada na fazenda Olhos D'Água na amostragem realizada por meio de cartões adesivos amarelos, se deve ao fato de que algumas espécies apresentaram um número de indivíduos capturados muito superior às demais; uma única espécie, $H$. spottii, foi responsável por $44,9 \%$ dos espécimes coletados nessa propriedade. 
Para que medidas efetivas de manejo da CVC possam ser adotadas na citricultura do Nordeste do Brasil, é de fundamental importância a identificação das espécies vetoras de $X$. fastidiosa nessa região. Este trabalho mostra que o cicadelíneo vetor $A$. citrina e outros três potenciais vetores, H. spottii, Cicadellini sp. 1 e A. flagellata, provavelmente desempenham um papel importante na disseminação de $X$. fastidiosa em pomares cítricos no Litoral Norte da Bahia. Assim, seria importante a avaliação futura quanto à capacidade e eficiência de transmissão de $X$. fastidiosa pelas espécies dominantes em pomares de citros, já que as mesmas são distintas daquelas avaliadas como vetoras no estado de São Paulo.

\section{Agradecimentos}

Aos proprietários das fazendas onde foram realizadas as coletas. À Profa Keti Zanol (UFPR) pelo auxílio na identificação taxonômica. Ao Prof Sinval Silveira Neto (ESALQ/USP) pelo auxilio na análise faunística. Aos Eng Agr Geraldo Almeida, Eng Agr Sandra Fontes, Tec Agr Francisco Nascimento (EBDA), Eng Agr Verônica Moitinho e Téc Agr Mário Cardoso (Secretaria da Agricultura de Rio Real) pela realização das coletas. Ao CNPq pela concessão de bolsa de produtividade ao segundo e último autor. À CAPES pela concessão de bolsa de mestrado ao primeiro autor e à EMBRAPA/CNPMF pelo apoio logístico na realização deste trabalho.

\section{Referências}

Atkinson A C (1985) Plots, transformations, and regression. New York, Oxford University Press, 282p.

De Negri J D (1990) Clorose variegada dos citros: nova anomalia afetando pomares em São Paulo e Minas Gerais. Comunicado técnico no 82, Ext Rural, CATI, Campinas, $6 \mathrm{p}$.

Giustolin T A, Lopes J R S, Querino R B, Cavichioli R, Zanol K, Azevedo Filho W S, Firmino D C, Mendes M A (2009) Diversidade de Hemiptera, Auchenorrhyncha em citros, café e fragmento de floresta nativa do estado de São Paulo. Neotrop Entomol 38: 834-841.

Kaloostian G H, Oldfield G N, Pierce H D, Calavan E C (1985) Spiroplasma citri and its transmission to citrus and other plants by leafhoppers, p.447-450. In Maramorosch K, Harris K F (eds) Leafhopper vectors and plant disease agentes. New York, Academic Press, 654p.

Laranjeira F F, Bergamin Filho A, Amorim L, Berger R D (1998) Aspectos práticos da epidemiologia da clorose variegada dos citros. Laranja 19: 79-90.

Lee R F, Beretta M J G, Hartung J H, Hooker M E, Derrick K S (1993) Citrus variegated chlorosis: confirmation of a Xylella fastidiosa as the causal agent. Summa Phytopathol 19: 123-125.

Lopes J R S (1999) Estudos com vetores de Xylella fastidiosa e implicações no manejo da clorose variegada dos citros. Laranja 20: $329-344$.
Lopes S A, Marcussi S, Torres S C Z, Souza V, Fagan C, França S C, Fernandes N G, Lopes J R S (2003) Weeds as alternative hosts of the citrus, coffee, and plum strains of Xylella fastidiosa in Brazil. Plant Dis 87: 543-549.

Mountford M D (1962) An index of similarity and its application to classificatory problems, p.43-50. In Murphy P W (ed) Prog. Soil Zoology, Butterworths, London, 398p.

Nielson M W (1985) Leafhoppers systematics, p.11-39. In Nault L R, Rodriguez J G (eds) The leafhoppers and planthoppers. New York, John Wiley, 500p.

Nunes W M C, Molina R O, Albuquerque F A, Corazza-Nunes M J, Zanutto C A, Machado M A (2007) Flutuação populacional de cigarrinhas vetoras de Xylella fastidiosa Wells et al. em pomares comerciais de citros no Noroeste do Paraná. Neotrop Entomol 36: 254-260.

Ott A P, Azevedo-Filho W S, Ferrari A, Carvalho G S (2006) Abundância e sazonalidade de cigarrinhas (Hemiptera, Cicadellidae, Cicadellinae) em vegetação herbácea de pomar de laranja doce, no município de Montenegro, estado do Rio Grande do Sul, Brasil. Iheringia, Sér Zool 96: 425-429.

Paiva P E B, Silva J L, Gravena S, Yamamoto P T (1996) Cigarrinhas de xilema em pomares de laranja do estado de São Paulo. Laranja 17: 41-54.

Purcell A H (1989) Homopteran transmission of xylem-inhabiting bacteria, p.243-266. In Harris K F (ed) Advances in disease vector research. New York, Springer-Verlag, 363p.

Purcell A H (1994) Cigarrinhas na cultura de citros, p.195-209. In Seminário internacional de citros - MIP, 3, Campinas, Fundação Cargill, 310p.

Redak R A, Purcell A H, Lopes J R S, Blua M J, Mizell III R F, Andersen P C (2004) The biology of xylem fluid - feeding insect vectors of Xylella fastidiosa and their relation to disease epidemiology. Annu Rev Entomol 49: 243- 270.

Roberto S R, Coutinho A, Lima J E O, Miranda V S, Carlos E F (1996) Transmissão de Xylella fastidiosa pelas cigarrinhas Dilobopterus costalimai, Acrogonia terminalis e Oncometopia facialis em citros. Fitopatol Bras 21: 517-518.

Roberto S R, Pria Jr W D, Yamamoto P T, Fellipe M R, Freitas E $P$ (2000) Espécies e flutuação populacional de cigarrinhas em viveiros de citros, em Gavião Peixoto (SP). Laranja 21: 65-79.

Santos Filho H P, Barbosa C J, Matrangolo W J R, Ribeiro J S, Meissner Filho P E, Miranda M P (1999) Ocorrência da clorose variegada dos citros (CVC) no estado da Bahia. Fitopatol Bras 24:190.

Silveira Neto S, Monteiro R C, Zucchi R A, Moraes R C B (1995) Uso da análise faunística de insetos na avaliação do impacto ambiental. Sci Agric 52: 9-15.

Silveira Neto S, Nakano O, Barbin D, Villa Nova NA(1976) Manual de ecologia dos insetos. Piracicaba, Agronômica Ceres, 416p.

Superintendência de Estudos econômicos e sociais da Bahia. Informações geoambientais http://www.sei.ba.gov.br/sei/ frame_tabela (Acessado em15 set. 2006). 
Teixeira D C, Wulff N A, Martins E C, Kitajima E W, Bassanezi R, Ayres A J, Eveillard S, Saillard C, Bové J M (2008) A phytoplasma closely related to the pigeon pea witches'-broom phytoplasma (16Sr IX) is associated with citrus huanglongbing symptoms in the state of São Paulo, Brazil. Phytopathology 98: 977-984.

Wilson M R, Claridge M F (1991) Handbook for the identification of leafhoppers of rice. Wallingford, C.A.B International, 142p.

Yamamoto P T, Gravena S (2000) Espécies e abundância de cigarrinhas e psilídeos (Homoptera) em pomares cítricos. An Soc Entomol Brasil 29: 169-176.

Yamamoto P T, Lopes J R S (2004) Cigarrinhas na proliferação da clorose variegada dos citros. Visão Agrícola 2: 60-63.

Yamamoto P T, Pria Jr W D, Roberto S R, Fellipe M R, Freitas E P (2001) Flutuação populacional de cigarrinhas (Hemiptera: Cicadellidae) em pomar cítrico em formação. Neotrop Entomol 30: $175-177$.
Yamamoto P T, Roberto S R, Pria Jr W D, Felippe M R, Miranda V S, Teixeira D C, Lopes J R S (2002) Transmissão de Xylella fastidiosa por cigarrinhas Acrogonia virescens e Homalodisca ignorata (Hemiptera:Cicadellidae) em plantas cítricas. Summa Phytopathol 28: 178-181.

Young D A (1968) Taxonomic study of the Cicadellinae. Part 1, Proconiini. U S Nat Mus Bull 261, 287p.

Young D A (1977) Taxonomic study of the Cicadellinae (Homoptera: Cicadellidae). Part 2, New World Cicadellini and genus Cicadella. U.S.D.A., Tech Bull 239, 1135p.

Received 22/XI/07. Accepted 12/II/09. 
Appendix 1 Índices faunísticos das principais espécies de cigarrinhas coletadas por cartões adesivos amarelos em pomares cítricos de três fazendas de Rio Real, BA, de março/2002 a fevereiro/2003. As letras em negrito no corpo da tabela representam as espécies predominantes de acordo com os índices faunísticos.

\begin{tabular}{|c|c|c|c|c|c|c|c|c|c|c|c|c|c|c|c|}
\hline \multirow{2}{*}{$\begin{array}{l}\text { Família } \\
\text { Subfamília } \\
\text { Espécie }\end{array}$} & \multicolumn{5}{|c|}{ Faz. Olhos D’Água } & \multicolumn{5}{|c|}{ Faz. Araújo } & \multicolumn{5}{|c|}{ Faz. Esperança } \\
\hline & $\mathrm{T}^{1}$ & $\mathrm{D}^{2}$ & $\mathrm{~A}^{3}$ & $\mathrm{~F}^{4}$ & $\mathrm{C}^{5}$ & $\mathrm{~T}^{1}$ & $\mathrm{D}^{2}$ & $\mathrm{~A}^{3}$ & $\mathrm{~F}^{4}$ & $\mathrm{C}^{5}$ & $\mathrm{~T}^{1}$ & $\mathrm{D}^{2}$ & $A^{3}$ & $\mathrm{~F}^{4}$ & $\mathrm{C}^{5}$ \\
\hline \multicolumn{16}{|l|}{ Aetalionidae } \\
\hline Aetalion sp. & 1 & ND & $\mathrm{r}$ & $\mathrm{PF}$ & $\mathrm{Z}$ & 1 & ND & $\mathrm{d}$ & $\mathrm{PF}$ & $\mathrm{Z}$ & 1 & ND & $\mathrm{r}$ & $\mathrm{PF}$ & $\mathrm{Z}$ \\
\hline \multicolumn{16}{|l|}{ Cicadellidae } \\
\hline \multicolumn{16}{|l|}{ Cicadellinae } \\
\hline Acrogonia citrina Mar. \& Cav. & 73 & SD & sa & SF & $\mathbf{W}$ & 10 & $\mathbf{D}$ & c & $\mathbf{F}$ & $\mathbf{Y}$ & 3 & ND & $\mathrm{d}$ & $\mathrm{PF}$ & $\mathrm{Z}$ \\
\hline Acrogonia flagellata Young & 69 & SD & sa & SF & $\mathbf{W}$ & 47 & $\mathbf{D}$ & ma & MF & $\mathbf{W}$ & 46 & $\mathbf{D}$ & ma & MF & $\mathbf{W}$ \\
\hline Cicadellini sp. 1 & 129 & SD & sa & SF & $\mathbf{W}$ & 49 & $\mathrm{D}$ & ma & MF & $\mathrm{W}$ & 41 & D & ma & MF & $\mathbf{W}$ \\
\hline Cicadellini sp. 2 & - & - & - & - & - & 1 & ND & $\mathrm{d}$ & $\mathrm{PF}$ & $\mathrm{Z}$ & - & - & - & - & - \\
\hline Crossogonalia hectica (Signoret) & 4 & ND & ma & MF & $\mathrm{Z}$ & 1 & ND & d & $\mathrm{PF}$ & $\mathrm{Z}$ & 1 & ND & r & $\mathrm{PF}$ & $\mathrm{Z}$ \\
\hline Diedrocephala variegata (Fabricius) & - & - & - & - & - & 1 & ND & d & $\mathrm{PF}$ & $\mathrm{Z}$ & - & - & - & - & - \\
\hline Diestostemma sp. & - & - & - & - & - & - & - & - & - & - & 1 & ND & r & $\mathrm{PF}$ & $\mathrm{Z}$ \\
\hline Erythrogonia dubia Medler & - & - & - & - & - & 1 & ND & $\mathrm{d}$ & $\mathrm{PF}$ & $\mathrm{Z}$ & - & - & - & - & - \\
\hline Homalodisca spottii Tak., Cav. \& Mck. & 301 & SD & sa & SF & $\mathbf{W}$ & 33 & $\mathbf{D}$ & ma & MF & $\mathbf{W}$ & 37 & $\mathbf{D}$ & ma & MF & $\mathbf{W}$ \\
\hline Oncometopia clarior (Walker) & - & - & - & - & - & 3 & ND & c & $\mathrm{F}$ & $\mathrm{Z}$ & 2 & ND & $\mathrm{r}$ & $\mathrm{PF}$ & $\mathrm{Z}$ \\
\hline Tapajosa fulvopuctata (Signoret) & 1 & ND & $\mathrm{r}$ & $\mathrm{PF}$ & $\mathrm{Z}$ & - & - & - & - & - & 3 & ND & d & $\mathrm{PF}$ & $\mathrm{Z}$ \\
\hline \multicolumn{16}{|l|}{ Deltocephalinae } \\
\hline Bahita sp. 1 & - & - & - & - & - & - & - & - & - & - & 29 & $\mathbf{D}$ & ma & MF & $\mathbf{W}$ \\
\hline Bahita sp. 2 & - & - & - & - & - & - & - & - & - & - & 10 & $\mathrm{D}$ & c & $\mathrm{F}$ & $\mathrm{Z}$ \\
\hline Scaphytopius sp. & 56 & SD & sa & SF & $\mathbf{W}$ & 9 & $\mathrm{D}$ & c & $\mathrm{F}$ & $\mathrm{Y}$ & 49 & $\mathbf{D}$ & ma & MF & W \\
\hline \multicolumn{16}{|l|}{ Membracidae } \\
\hline Bobonata sp. & 3 & ND & c & $\mathrm{F}$ & $\mathrm{Z}$ & - & - & - & - & - & 4 & ND & d & $\mathrm{PF}$ & $\mathrm{Z}$ \\
\hline Ceresa ustulata Fairmaire & 7 & $\mathrm{D}$ & ma & $\mathrm{MF}$ & $\mathrm{Y}$ & 21 & D & ma & MF & $\mathbf{W}$ & 6 & $\mathrm{D}$ & C & $\mathrm{F}$ & $\mathrm{Z}$ \\
\hline Cyphonia clavigera (Fabricius) & 3 & ND & c & $\mathrm{F}$ & $\mathrm{Z}$ & - & - & - & - & - & 2 & ND & $\mathrm{r}$ & $\mathrm{PF}$ & $\mathrm{Z}$ \\
\hline Enchenopa sp. & 2 & ND & c & $\mathrm{F}$ & $\mathrm{Z}$ & - & - & - & - & - & 4 & ND & d & $\mathrm{PF}$ & $\mathrm{Z}$ \\
\hline Enchophyllum sp. & 2 & ND & c & $\mathrm{F}$ & $\mathrm{Z}$ & 2 & ND & d & $\mathrm{PF}$ & $\mathrm{Z}$ & - & - & - & - & - \\
\hline Entylia sp. & 2 & ND & c & $\mathrm{F}$ & $\mathrm{Z}$ & 3 & ND & C & $\mathrm{F}$ & $\mathrm{Z}$ & - & - & - & - & - \\
\hline Hygris sp. & - & - & - & - & - & 1 & ND & d & $\mathrm{PF}$ & $\mathrm{Z}$ & - & - & - & - & - \\
\hline Membracis sp. & - & - & - & - & - & 3 & ND & c & $\mathrm{F}$ & $\mathrm{Z}$ & 7 & $\mathrm{D}$ & C & $\mathrm{F}$ & $\mathrm{Z}$ \\
\hline
\end{tabular}

${ }^{1}$ Total de espécimes coletados em 20 avaliações quinzenais; ${ }^{2}$ Dominância - SD: super dominante; D: dominante; ND: não dominante; ${ }^{3}$ Abundância - sa: super abundante; ma: muito abundante; a: abundante; c: comum; d: dispersa; r: rara; ${ }^{4}$ Frequência - SF: super frequente; MF: muito frequente; F: frequente; PF: pouco frequente; ${ }^{5} \mathrm{Constância}$ - W: constante; Y: acessória; Z: acidental 
Appendix 2 Índices faunísticas das principais espécies de cigarrinhas coletadas com rede de varredura na vegetação rasteira de pomares cítricos de três fazendas localizadas em Rio Real, BA, no período de março/2002 a fevereiro/2003. As letras em negrito no corpo da tabela representam as espécies predominantes de acordo com os índices faunísticos.

\begin{tabular}{|c|c|c|c|c|c|c|c|c|c|c|c|c|c|c|c|}
\hline \multirow{2}{*}{$\begin{array}{l}\text { Família } \\
\text { Subfamília } \\
\text { Espécie }\end{array}$} & \multicolumn{5}{|c|}{ Faz. Olhos D’Água } & \multicolumn{5}{|c|}{ Faz. Araújo } & \multicolumn{5}{|c|}{ Faz. Esperança } \\
\hline & $\mathrm{T}^{1}$ & $\mathrm{D}^{2}$ & $\mathrm{~A}^{3}$ & $\mathrm{~F}^{4}$ & $\mathrm{C}^{5}$ & $\mathrm{~T}^{1}$ & $\mathrm{D}^{2}$ & $A^{3}$ & $\mathrm{~F}^{4}$ & $\mathrm{C}^{5}$ & $\mathrm{~T}^{1}$ & $\mathrm{D}^{2}$ & $A^{3}$ & $\mathrm{~F}^{4}$ & $\mathrm{C}^{5}$ \\
\hline \multicolumn{16}{|l|}{ Cicadellidae } \\
\hline \multicolumn{16}{|l|}{ Cicadellinae } \\
\hline Bucephalogonia xanthophis (Berg) & 1 & ND & $\mathrm{r}$ & $\mathrm{PF}$ & $\mathrm{Z}$ & - & - & - & - & - & - & - & - & - & - \\
\hline Erythrogonia dubia Medler & - & - & - & - & - & 3 & ND & C & $\mathrm{F}$ & $\mathrm{Z}$ & 13 & $\mathrm{D}$ & c & $\mathrm{F}$ & $\mathrm{W}$ \\
\hline Ferrariana trivittata (Signoret) & - & - & - & - & - & 3 & ND & C & $\mathrm{F}$ & $\mathrm{Z}$ & - & - & - & - & - \\
\hline Homalodisca spottii Tak. Cav. \& Mck & - & - & - & - & - & 1 & ND & $\mathrm{d}$ & $\mathrm{PF}$ & $\mathrm{Z}$ & - & - & - & - & - \\
\hline Hortensia similis (Walker) & 8 & $\mathrm{D}$ & C & $\mathrm{F}$ & $\mathrm{Y}$ & 44 & SD & sa & SF & $\mathbf{W}$ & 18 & $\mathbf{D}$ & ma & MF & $\mathbf{W}$ \\
\hline \multicolumn{16}{|l|}{ Deltocephalinae } \\
\hline Acinopterus gentilis (Berg) & 19 & D & ma & MF & $\mathbf{Y}$ & - & - & - & - & - & - & - & - & - & - \\
\hline Stirellus bicolor (Van Duzee) & 2 & ND & r & $\mathrm{PF}$ & $\mathrm{Z}$ & - & - & - & - & - & 3 & ND & c & $\mathrm{F}$ & $\mathrm{Z}$ \\
\hline \multicolumn{16}{|l|}{ Ledrinae } \\
\hline Xerophloea viridis (Fabricius) & - & - & - & - & - & - & - & - & - & - & 3 & ND & C & $\mathrm{F}$ & $\mathrm{Z}$ \\
\hline \multicolumn{16}{|l|}{ Typhlocybinae } \\
\hline Empoasca sp. & 19 & $\mathbf{D}$ & ma & MF & $\mathbf{Y}$ & 12 & $\mathrm{D}$ & ma & MF & $\mathrm{Y}$ & 22 & $\mathrm{D}$ & ma & $\mathrm{MF}$ & $\mathrm{Y}$ \\
\hline \multicolumn{16}{|l|}{ Cercopidae } \\
\hline Mahanarva sp. & - & - & - & - & - & - & - & - & - & - & 1 & ND & $\mathrm{d}$ & $\mathrm{PF}$ & $\mathrm{Z}$ \\
\hline Zulia sp. & - & - & - & - & - & 2 & ND & c & $\mathrm{F}$ & $\mathrm{Z}$ & - & - & - & - & - \\
\hline \multicolumn{16}{|l|}{ Membracidae } \\
\hline Ceresa ustulata Fairmaire & 14 & $\mathrm{D}$ & $\mathrm{a}$ & MF & $\mathrm{Y}$ & 27 & SD & sa & $\mathrm{SF}$ & $\mathrm{W}$ & - & - & - & - & - \\
\hline Cyphonia clavigera (Fabricius) & 2 & ND & r & $\mathrm{PF}$ & $\mathrm{Z}$ & - & - & - & - & - & - & - & - & - & - \\
\hline Tapinobolus sp. & 3 & ND & $\mathrm{d}$ & $\mathrm{PF}$ & $\mathrm{Z}$ & - & - & - & - & - & - & - & - & - & - \\
\hline
\end{tabular}

${ }^{1}$ Total de espécimes coletados em 13 avaliações; ${ }^{2}$ Dominância - SD: super dominante; D: dominante; ND: não dominante; ${ }^{3}$ Abundância - sa: super abundante; ma: muito abundante; a: abundante; c: comum; d: dispersa; r: rara; ${ }^{4}$ Frequência - SF: super frequente; MF: muito frequente; F: frequente; PF: pouco frequente; ${ }^{5}$ Constância - W: constante; Y: acessória; Z: acidental 